\title{
"Real-world" effectiveness of smoking cessation treatments: a population study
}

\section{Revision 2 based on second round of reviewer comments from Addiction}

Daniel Kotz PhD ${ }^{1,2^{*}}$, Jamie Brown $\mathrm{PhD}^{1}$, Robert West $\mathrm{PhD}^{1}$

${ }^{1}$ Department of Family Medicine, CAPHRI School for Public Health and Primary Care, Maastricht University Medical Centre, Maastricht, the Netherlands. ${ }^{2}$ Cancer Research UK Health Behaviour Research Centre, University College London, WC1E 6BT, UK

Running head: Smoking cessation treatment in the real world

Number of words: 4,008 (main text); 296 (abstract)

Tables: 4; Figures: 0

Supplementary Table: 1

Key words: smoking cessation, varenicline, bupropion, nicotine replacement therapy, behavioural support

${ }^{*}$ Correspondence to: Dr. Daniel Kotz, Department of Family Medicine, CAPHRI School for Public Health and Primary Care, Maastricht University Medical Centre, PO Box 616, 6200 MD Maastricht, the Netherlands. E-mail: d.kotz@maastrichtuniversity.nl, web: www.daniel-kotz.de, phone: +3143 3882893, FAX: +31 433619344 
Statement of competing interests: RW has undertaken research and consultancy and received travel funds from companies that develop and manufactures smoking cessation medications. He has a share of a patent for a novel nicotine delivery device. He is a trustee of the stop-smoking charity, QUIT and Co-direct of the National Centre for Smoking Cessation and Training. DK has received an unrestricted research grant from Pfizer for a smoking cessation trial. JB has received an unrestricted research grant from Pfizer. This study is partly funded by Pfizer under an investigator initiated award. 


\section{ABSTRACT}

\section{Aims}

There is a need for more evidence on the 'real-world' effectiveness of commonly used aids to smoking cessation from population-level studies. This study assessed the association between abstinence and use of different smoking cessation treatments after adjusting for key potential confounding factors.

\section{Design}

Cross-sectional data from aggregated monthly waves of a household survey: the Smoking Toolkit Study.

\section{Setting}

England.

\section{Participants}

10,335 adults who smoked within the previous 12 months and made at least one quit attempt during that time.

\section{Measurements}

Participants were classified according to their use of cessation aids in their most recent quit attempt: (1) medication (nicotine replacement therapy, bupropion, or varenicline) in combination with specialist behavioural support delivered by a National Health Service Stop Smoking Service; 
(2) medication provided by the prescribing health care professional without specialist behavioural support; (3) nicotine replacement therapy (NRT) bought over-the-counter; (4) none of these. The main outcome measure was self-reported abstinence up to the time of the survey, adjusted for key potential confounders including tobacco dependence.

\section{Findings}

Compared with smokers using none of the cessation aids, the adjusted odds of remaining abstinent up to the time of the survey were $3.25(95 \% \mathrm{Cl}=2.05-5.15)$ greater in users of prescription medication in combination with specialist behavioural support, $1.61(95 \% \mathrm{Cl}=1.33-1.94)$ greater in users of prescription medication combined with brief advice, and $0.96(95 \% \mathrm{Cl}=0.81-1.13)$ in users of NRT bought over-the-counter.

\section{Conclusions}

After adjusting for major confounding variables such as tobacco dependence, smokers in England who use a combination of behavioural support and pharmacotherapy in their quit attempts have almost three times the odds of success than those who use neither pharmacotherapy or behavioural support. Smokers who buy nicotine replacement therapy over the counter with no behavioural support have similar odds of success as stopping as those who stop without any aid. 


\section{INTRODUCTION}

The World Health Organization (WHO) estimates that smoking kills nearly six million people each year. ${ }^{1}$ Every year that someone continues to smoke after early middle age loses them 3 months of life expectancy. ${ }^{2}$ It is therefore important that every quit attempt has the best possible chance of success. There is strong evidence from multiple randomized controlled trials that behavioural support and several medications improve the success of quit attempts. ${ }^{3-9}$ However, populationbased studies about the effectiveness of smoking cessation treatments in the "real world" have produced mixed results. This is particularly important because we are now in the implementation phase of Article 14 of the WHO Framework Convention on Tobacco Control which mandates signatory countries to promote smoking cessation in their populations ${ }^{10,11}$; so real-world evidence on effectiveness of treatment to aid cessation will have global impact. Real-world studies using observational designs cannot provide the same degree of confidence in causal associations as randomised trials because of the possibility of residual confounding but without them the generalizability of the randomised trial evidence will always be called into question. Thus both types of study are essential.

Some "real world" studies have reported a lower chance of successful quitting in smokers who used nicotine replacement therapy (NRT) or bupropion than in smokers who tried to quit without medication. ${ }^{12-15}$ However, these studies did not adequately control for important confounding factors, most notably the fact that smokers who use these medications are more dependent on cigarettes. ${ }^{16-19}$ Of the few studies that have attempted to control for such confounding, one US study ${ }^{20}$ found a lower chance of successful quitting in users of NRT and bupropion compared with non-users of smoking cessation treatment whereas two multinational studies ${ }^{18,21}$ found higher chances of quitting in users of NRT, bupropion, or varenicline. However, these did not investigate 
the effect of behavioural support provided or distinguish between NRT bought over-the-counter versus obtained from a health care professional. This leaves a critical gap in the literature.

The current study is the first with adequate power to assess the real-world effectiveness of medication for smoking cessation combined with behavioural support in comparison with unaided quitting using population-based survey data while adjusting for key potential confounding factors. In addition to controlling for dependence, the current study adjusted for a number of other factors that have been associated with both successful quitting and choice of treatment including age, sex, social grade, and previous quit attempts. ${ }^{20,22-26}$ Importantly, this study also distinguishes between the provision of specialist behavioural support and brief advice. England is a country with the most extensive and comprehensive coverage of behavioural support and medications in the world, and the highest rate of use of these aids to cessation. ${ }^{27}$ Therefore it is probably the only country where a population level study of this kind could be undertaken. Every smoker has ready access to behavioural support and medication that is either free or available at nominal charge. In addition, all the forms of NRT are available to be purchased over-the-counter. As these aids have been available for at least 10 years, the market is mature and any associations are not likely to reflect the fact that the interventions are novel. This makes England a unique environment for the assessment of the real-world effectiveness of different quitting methods.

\section{METHODS}

We used data from the "Smoking Toolkit Study": an ongoing research program designed to provide information about smoking cessation and factors that promote or inhibit it at a population level. ${ }^{28,29}$ Each month a new sample of approximately 1,800 people aged 16 and over completes a face-to-face computer-assisted survey, of whom approximately 450 are smokers. The methods 
have been described in full elsewhere and have been shown to result in figures for key variables such as smoking prevalence that are nationally representative. ${ }^{28}$

\section{Study population}

For the current study, we used aggregated data from respondents to the survey in the period from November 2006 (the start of the survey) to May 2012 (the latest wave of the survey for which data were available), who smoked cigarettes (including hand-rolled) or any other tobacco product (e.g., pipe or cigar) daily or occasionally at the time of the survey or during the preceding 12 months. We included those who made at least one quit attempt in the preceding 12 months, assessed by asking: "How many serious attempts to stop smoking have you made in the last 12 months? By serious attempt I mean you decided that you would try to make sure you never smoked again. Please include any attempt that you are currently making and please include any successful attempt made within the last year." We also asked how long ago the most recent quit attempt started and categorised respondents into those who started their quit attempt in the last week or up to 6 months ago and those who started their quit attempt more than 6 months ago.

\section{Measurement of effect: use of smoking cessation treatments}

The use of smoking cessation treatments was assessed only for the most recent quit attempt and included: (1) NRT on prescription (NRT Rx), bupropion, or varenicline in combination with specialist behaviour support (i.e., one-to-one or group behavioural support delivered by a National Health Service (NHS) Stop Smoking Service); (2) NRT Rx, bupropion, or varenicline in combination with brief advice (delivered by the prescribing health care professional); (3) NRT bought over-thecounter; (4) none of these. 


\section{Measurement of outcome: self-reported non-smoking}

Our primary outcome was self-reported non-smoking up to the time of the survey. Respondents were asked: "How long did your most recent serious quit attempt last before you went back to smoking?". Those responding "I am still not smoking" were defined as non-smokers. Previous research has shown that self-reported abstinence in surveys of this kind is not subject to the kind of biases observed in clinical trials where there is social pressure to claim abstinence. ${ }^{30,31}$

\section{Measurement of potential confounders}

We measured variables potentially associated with the use of smoking cessation treatments and that may also have an effect on the outcome. These potential confounders were chosen a priori. The most important factor was cigarette dependence for which we used two questions. First, time spent with urges to smoke was assessed by asking: "How much of the time have you felt the urge to smoke in the past 24 hours? Not at all (coded 1), a little of the time (2), some of the time (3), a lot of the time (4), almost all of the time (5), all of the time (6)". Second, strength of urges to smoke was measured by asking "In general, how strong have the urges to smoke been?": slight (1), moderate (2), strong (3), very strong (4), extremely strong (5). This question was coded "0" for smokers who responded "not at all" to the previous question. These two ratings have been found in this population to be a better measure of dependence (more closely associated with relapse following a quit attempt) than other measures. ${ }^{32}$ Demographic characteristics we took into account were age, sex, and social grade (measured on an ordinal scale: $A B=$ managerial and professional occupations, $\mathrm{C} 1$ = intermediate occupations, $\mathrm{C} 2=$ small employers and own account workers, $\mathrm{D}=$ lower supervisory and technical occupations, and $\mathrm{E}=$ semi-routine and routine occupations, never workers, and long-term unemployed). Furthermore, we measured the number 
of quit attempts in the last year prior to the one in question, and time since the quit attempt in question was initiated.

\section{Data analyses}

Simple associations between potential confounders and use of the smoking cessation treatments were assessed with ANOVA for continuous variables and Pearson's $\chi^{2}$ for categorical variables. Tukey's post-hoc procedure was used for multiple comparisons of the two measures of tobacco dependence.

Our measure of dependence (strength of urges to smoke) assumed that the score relative to other smokers would stay the same from pre- to post-quitting. Thus a measure taken after the quit attempt would reflect, relative to other smokers in the same position (i.e., having stopped or failed to stop), what it would have been prior to it. The absolute score would reduce between these two occasions but this reduction would not be affected substantially by the method of quitting. If a method of quitting reduced strength of urges to smoke more than another method, this would tend to underestimate the effectiveness of that intervention because the smokers using this method would appear to be less dependent. If it increased the strength of urges it would overestimate the method's effectiveness by making it seem that the smokers were more dependent than they actually were. To test for this bias we examined in an ANCOVA whether the difference in strength of urges to smoke in smokers versus quitters varied as a function of the method of quitting, adjusting for the time since the quit attempt started. Although the power to detect such an interaction in the population would be relatively low, our interest is only in whether the interaction exists in this sample since it is that which could artificially inflate or deflate our estimate of the association between quitting method and success. 
For our primary analysis, we used a multiple logistic regression model in which we regressed the outcome measure (self-reported non-smoking compared with smoking) on the effect measure (use of each of the three smoking cessation treatments compared with no use of such treatments), adjusted for the above mentioned confounders and year of the survey. We also included two interaction terms: (1) between time since last quit attempt and time spent with urges, and (2) between time since last quit attempt and strength of urges to smoke. These interaction terms were used to account for the fact that urges to smoke following the quit attempt will be influenced by whether the respondent is currently abstinent and the duration of abstinence. However, we also ran this model after excluding the two interaction terms in a sensitivity analysis.

The sample size in our study provided $99 \%$ power to detect an odds ratio of 3.0 for the comparison of medication on prescription + specialist behavioural support versus no treatment, and $94 \%$ power to detect an odds ratio of 1.5 for the comparison of medication on prescription + brief advice versus no treatment (effect sizes estimated from randomised controlled trials).

In addition to the model from the primary analysis ("fully adjusted model"), we constructed a simple model including only the effect measure ("unadjusted model") and a model that included the effect measure, year of the survey and all confounders except for the two measures of tobacco dependence and their interaction terms ("partially adjusted model") to show the extent of confounding effects of tobacco dependence. 
In a sensitivity analysis we excluded respondents who had used telephone counselling for smoking cessation during their most recent quit attempt; very few smokers in England use this form of treatment so it is not possible to assess its association with abstinence. In the primary analysis these smokers were conservatively counted in the "no treatment" group unless they had also used medication whereas in the sensitivity analysis they were excluded from the analysis.

All analyses were repeated in the two subsamples of respondents who had started their most recent quit attempt less versus more than 6 months ago in order to assess the occurrence of differential recall bias. It has been suggested that smokers who try to stop unaided forget failed quit attempts more quickly than those who use treatment. ${ }^{16}$ In the presence of such bias the longterm effectiveness of smoking cessation treatments would be underestimated. However, a positive association would provide evidence for lasting treatment effects of a kind that have been questioned by previous researchers. ${ }^{12}$

All analyses were performed with complete cases. Respondents with missing data on one or more of the variables were excluded (5.5\% of the initial sample).

\section{RESULTS}

The study population consisted of 10,335 respondents; 8,932 (86.4\%) who smoked and 1,403 (13.6\%) who were abstinent at the time of the survey. The unadjusted abstinence rates were $19.1 \%(\mathrm{~N}=39)$ for users of medication on prescription in combination with specialist behavioural support, $15.2 \%(\mathrm{~N}=259)$ for users of prescription medication combined with brief advice, $10.2 \%$ $(\mathrm{N}=322)$ for users of NRT bought over-the-counter, and $14.8 \%(\mathrm{~N}=783)$ for those using none of these treatments. A subgroup of 6,510 respondents ( $63.0 \%$ of the full study population) had 
started their last quit attempt less than 6 months ago and 3,825 (37.0\%) had started their last quit attempt more than 6 months ago. Demographic and smoking-related characteristics of the full sample are shown in Table 1. The characteristics of the two subsamples of smokers who had started their last quit attempt more versus less than 6 months ago were similar (not shown in the table).

A total of 1,910 respondents (18.5\%) had used some form of prescription medication during their most recent quit attempt. Among these respondents, the majority had used NRT Rx $(58.1 \%$, $\mathrm{N}=1,110)$, followed by varenicline $(28.2 \%, \mathrm{~N}=538)$, and bupropion $(10.9 \%, \mathrm{~N}=208)$. The remaining $2.8 \%(\mathrm{~N}=54)$ of respondents had used some combination of these medications.

The use of treatments was associated with age, sex, time since last quit attempt started and the two measures of dependence (time spent with and strength of urges to smoke) (Table 2). The use of treatments also differed according to social grade. The post-hoc comparisons showed more time spent with urges to smoke and stronger urges to smoke in the three groups that used smoking cessation medication compared with the group that did not use medication (all $p<0.001$ ).

Table 3 shows the differences in strength of urges to smoke in smokers versus non-smokers, stratified by method of quitting. As would be expected strengths of urges to smoke were higher in smokers than in quitters and in those smokers using more intensive methods of quitting. However, the mean differences in strength of urges between smokers and quitters were not different across the methods of quitting: The interaction term between smoking status (smokers versus quitters) and method of quitting in the ANCOVA of the strength of urges adjusted for the time since quit attempt started was not statistically significant $(p=0.44)$. 
There was evidence of preferential recall of quit attempts made $6+$ months ago if medication on prescription or behavioural support was used but not if NRT bought over-the-counter was used. Thus, reported rates of use of medication in combination with specialist behavioural support and use of prescription medication combined with brief advice were higher in respondents who had started their most recent quit attempt more than 6 months ago compared with the subsample who had started less than 6 months ago (2.5\% vs. $1.7 \%$ for medication combined with specialist behavioural support and $17.7 \%$ vs. $15.8 \%$ for medication combined with brief advice, $p<0.01$ ). Reported use of NRT over-the-counter was similar within the subsamples (29.9\% vs. $30.5 \%)$, whereas the reported rate of no treatment use was lower within the subsample of respondents who had started their most recent quit attempt more than 6 months ago than in the subsample who had started less than 6 months ago (49.9\% vs. $52.1 \%, p<0.01)$.

Table 4 shows that in the full sample, the fully adjusted odds (model 4) of non-smoking in users of medication on prescription in combination with specialist behavioural support were 3.25 times higher compared with the no-treatment group. The odds were 2.02 times higher compared with the group that used prescription medication combined with brief advice (not shown in the table). In the latter group, the odds were 1.61 times higher compared with the no-treatment group. The use of NRT bought over-the-counter was not associated with abstinence (OR=0.96). These odds ratios were similar to the odds ratios of the fully adjusted model excluding the two interaction terms (model 3). The relative magnitudes of the odds ratios from the fully adjusted model (model 4) with the unadjusted model (model 1 ) and the partially adjusted model 2 show the large confounding effects of cigarette dependence. 
A total of 114 respondents (1.1\%) reported having used telephone counselling during their most recent quit attempt. The percentage of telephone counselling users was higher in the group that used medication on prescription in combination with NHS counselling $(7.4 \%)$ than in the other two treatment groups and the no-treatment group (percentages between 0.6-1.2\%). Excluding these respondents from the primary analysis increased the association between non-smoking and use of medication on prescription in combination with specialist behavioural support (fully adjusted $\mathrm{OR}=3.51,95 \% \mathrm{Cl}=2.19-5.61)$, but did not change the association with the other two treatments.

In smokers who started their quit attempt more than 6 months ago, the fully adjusted odds of non-smoking in users of medication on prescription in combination with specialist behavioural support were $2.32(95 \% \mathrm{Cl}=1.15-4.67)$ times higher compared with the no treatment group, whereas the odds were not statistically significantly higher in users of prescription medication combined with brief advice (OR=1.26, 95\%Cl=0.91-1.76).

\section{DISCUSSION}

Use of prescription medication in combination with specialised behavioural support during attempts to quit smoking was associated with the success of such attempts as was use of prescription medication with limited support. No such association was detected for NRT bought over-the-counter.

Our adjusted odds ratio of 1.61 in users of prescription medication combined with brief advice compared with non-users of treatment was similar to that from meta-analyses of randomized placebo-controlled trials ${ }^{6,7,8}$. Our estimated effectiveness of adding behavioural support to medication ( $\mathrm{OR}=2.02$ ) was slightly higher than would be expected from a meta-analysis performed 
for the US guidelines. ${ }^{33}$ It is noteworthy that adjusting for dependence made a substantial difference to these odds ratios and emphasises the importance in this kind of study of adequately controlling for this very substantial confounder.

Our findings conflicted with those from a meta-analysis of the effectiveness of NRT bought overthe-counter. ${ }^{34}$ Preferential recall of quit attempts using this method does not appear to explain this finding. We cannot rule out an effect of unmeasured confounding factors but it should be noted that this ought to have undermined the observed effects of behavioural support and medication on prescription, yet we were able to detect these effects. If NRT over-the-counter has become ineffective in England, this represents a considerable financial and opportunity cost for smokers and steps need to be taken urgently to address this.

As noted in the introduction, findings from similar studies to ours without adequate adjustment for cigarette dependence ${ }^{12-15,20}$ cannot be relied upon. This rules out most cross-sectional surveys because the most commonly used measure of cigarette dependence uses number of cigarettes smoked and time to first cigarette of the day. When smokers relapse they tend to do so with reduced consumption which can lead to a false estimation of prior dependence. We avoided this by using a validated measure involving ratings of current urges to smoke and statistically adjust for whether this was during normal smoking or a period of abstinence. ${ }^{32}$ However, our findings with regard to medication are consistent with many prospective real-world studies. ${ }^{18,21,25,35-38}$

Studies of the kind reported here do not in themselves allow causal inferences of the association between treatment and outcome but they are essential to examine how far the findings from randomized trials generalize to population samples. We reduced the risk of confounding further 
than any previous study by adjusting for cigarette dependence, age, sex, social grade, and previous quit attempts. However, residual confounding may have occurred as not all factors associated with self-selection of treatment were measured in our survey, such as co-morbidity ${ }^{39}$ or psychological distress ${ }^{40}$. Motivation to quit may also be positively associated with both use of treatment and success. However, population studies have generally not found an association between motivation to quit and success of quit attempts. ${ }^{41}$ Finally, our survey is limited by the fact that it does not contain data on medication adherence.

The value of ratings of strength of urges to smoke as a measure of dependence in cross-sectional research would have been reduced if different methods of stopping had been found differentially to be linked to lower or higher levels of urges in abstinent smokers. For example, a method of stopping that led to a relatively higher reduction in urges might underestimate the effectiveness of that method by making it seem that those using it were less dependent. However, we did not find evidence in this population data set that urges to smoke in smokers versus quitters differed as a function of method. It is very unlikely, therefore, that our dependence measure led to substantial overestimation or underestimation of the effectiveness of the different methods.

Reliance on recall is inevitable in population studies of this kind and even in prospective studies it is an issue unless one stimulates quit attempts. In our study, with the quit attempt having occurred up to 12 months ago, the scope for recall bias is significant. ${ }^{20,42}$ This would tend to reduce the ability to detect an effect and does not undermine the finding of a significant benefit of behavioural support plus medication. The effect sizes for medication with specialist behavioural support or with brief advice were lower in smokers who started their quit attempt more than 6 months ago than in smokers who started their quit attempt less than 6 ago. This finding may be a 
result of differential recall bias. We found some evidence in our study that the use of prescription medication during a quit attempt, especially when combined with specialised behavioural support, was recalled better than no use of treatment during a quit attempt (we did not find evidence of recall bias in usage of NRT bought over-the-counter). Our finding may, however, also be a result of reduced long-term effectiveness of prescription medication when prescribed with brief advice only.

To maximize statistical power, we combined the prescription of NRT, bupropion, and varenicline in our study. It would be useful to compare these medications with each other once sufficient samples have been accumulated.

We defined our measure of outcome as whether or not participants had remained abstinent from the quit date to the time of the survey. Another approach would have been to assess how long participants reported having been abstinent since their quit date, even if they had relapsed by the time of the survey. We decided not to use this measure because of added noise and potential bias with smokers recalling the point at which they had relapsed, bearing in mind that they make different interpretations on what constituted relapse (e.g. was it the first lapse, or return to daily smoking?). It was not feasible in our large population study to biochemically validate self-reported non-smoking. This would be a serious limitation in randomized controlled trials because of the possibility of differential likelihood of falsely claiming abstinence by participants in the active treatment. ${ }^{43}$ However, in population surveys the misreporting rate is low. ${ }^{30,31}$ A major strength of our study is the use of a very large, representative sample of the English population - sufficient to permit detection of an effect of behavioural support despite its low 
frequency. Our study included all smokers aged 16 years or older including those who smoke less than 10 cigarettes per day, a group that constitutes one third of current smokers. ${ }^{44}$ Furthermore, we used aggregated data from monthly surveys over a period of 5.5 years and therefore eliminated potential bias from the fact that the rate of attempts to quit in smokers is different at different times of the year.

\section{Conclusions and recommendations}

This is the first evidence from a population sample of the real-world effectiveness of the combination of behavioural support and stop-smoking medication as recommended by the US Department of Health and Human Services guidelines ${ }^{33}$ once adequate adjustment is made for confounding particularly by cigarette dependence. We also confirmed the effectiveness of stopsmoking medication provided with minimal support by health professionals, at least in the short term. Importantly, we did not detect an effect of NRT bought over-the-counter.

Health care professionals should know that smokers who seek treatment differ from smokers who try to quit unaided in that they have more difficulties quitting. In those smokers, a combination of evidence-based medication combined with expert behavioural support is recommended. More research is urgently needed on real world effectiveness of nicotine replacement therapy bought over the counter.

\section{FUNDING}

The Smoking Toolkit Study is funded by the English Department of Health, Cancer Research UK, Pfizer, GlaxoSmithKline, and Johnson and Johnson. Pfizer, Johnson and Johnson, and GlaxoSmithKline are manufacturers of smoking cessation products who had no involvement in the 
design of the study, collection, analysis or interpretation of the data, the writing of the report, or the decision to submit the paper for publication.

\section{CONTRIBUTORSHIP STATEMENT}

RW designed the Smoking Toolkit Study and outlined the manuscript. DK analysed the data for this manuscript and wrote the first full draft of the manuscript. All authors contributed to the writing of subsequent versions and approved the final version. 


\section{REFERENCE LIST}

1. World Health Organisation (WHO). WHO report on the global tobacco epidemic. Warning about the dangers of tobacco. Geneva2011.

2. Doll R, Peto R, Boreham J, Sutherland I. Mortality in relation to smoking: 50 years' observations on male British doctors. BMJ (Clinical research ed. 2004;328(7455):15191510.

3. Lancaster T, Stead Lindsay F. Individual behavioural counselling for smoking cessation. Cochrane Database of Systematic Reviews. 2005(2).

4. Stead L, Bergson G, Lancaster T. Physician advice for smoking cessation. Cochrane database of systematic reviews (Online). 2008(2).

5. Stead LF, Lancaster T. Group behaviour therapy programmes for smoking cessation. Cochrane Database of Systematic Reviews. 2005(2).

6. Stead LF, Perera R, Bullen C, Mant D, Lancaster T. Nicotine replacement therapy for smoking cessation. Cochrane Database of Systematic Reviews. 2008(1).

7. Hughes JR, Stead LF, Lancaster T. Antidepressants for smoking cessation. Cochrane Database of Systematic Reviews. 2007(1).

8. Cahill K, Stead Lindsay F, Lancaster T. Nicotine receptor partial agonists for smoking cessation. Cochrane Database of Systematic Reviews. 2012(4).

9. Stead Lindsay F, Lancaster T. Combined pharmacotherapy and behavioural interventions for smoking cessation. Cochrane Database of Systematic Reviews. 2012(10).

10. Raw M. Framework Convention on Tobacco Control (FCTC) Article 14 guidelines: a new era for tobacco dependence treatment. Addiction (Abingdon, England). 2011;106(12):20552057. 
11. World Health Organisation (WHO). WHO Framework on Tobacco Control. Available at: http://whqlibdoc.who.int/publications/2003/9241591013.pdf [accessed 6 September 2012]. Geneva: WHO;2005.

12. Pierce JP, Gilpin EA. Impact of Over-the-Counter Sales on Effectiveness of Pharmaceutical Aids for Smoking Cessation. JAMA. 2002;288(10):1260-1264.

13. Lee CW, Kahende J. Factors associated with successful smoking cessation in the United States, 2000. American journal of public health. Aug 2007;97(8):1503-1509.

14. Hagimoto A, Nakamura M, Morita T, Masui S, Oshima A. Smoking cessation patterns and predictors of quitting smoking among the Japanese general population: a 1-year follow-up study. Addiction (Abingdon, England). 2010;105(1):164-173.

15. Yang J, Hammond D, Driezen $P$, et al. The use of cessation assistance among smokers from China: Findings from the ITC China Survey. BMC public health. 2011;11(1):75.

16. Borland R, Partos TR, Cummings KM. Systematic Biases in Cross-sectional Community Studies may Underestimate the Effectiveness of Stop-Smoking Medications. Nicotine \& Tobacco Research. February 7, 20122012 Feb 7. [Epub ahead of print].

17. Shiffman S, Di Marino ME, Sweeney CT. Characteristics of selectors of nicotine replacement therapy. Tobacco control. Oct 2005;14(5):346-355.

18. West R, Zhou X. Is nicotine replacement therapy for smoking cessation effective in the "real world"? Findings from a prospective multinational cohort study. Thorax. Nov 2007;62(11):998-1002.

19. Hughes JR, Peters EN, Naud S. Effectiveness of Over-the-Counter Nicotine Replacement Therapy: A Qualitative Review of Nonrandomized Trials. Nicotine \& Tobacco Research. July 1, $20112011 ; 13(7): 512-522$. 
20. Shiffman S, Brockwell SE, Pillitteri JL, Gitchell JG. Use of Smoking-Cessation Treatments in the United States. American journal of preventive medicine. 2008;34(2):102-111.

21. Kasza KA, Hyland AJ, Borland R, et al. Effectiveness of Stop-Smoking Medications: Findings from the International Tobacco Control (ITC) Four Country Survey. Addiction (Abingdon, England). 2013;108(1):193-202.

22. Kotz D, Fidler J, West R. Factors associated with the use of aids to cessation in English smokers. Addiction (Abingdon, England). Jun 22 2009;104:1403-1410.

23. Kotz D, West R. Explaining the social gradient in smoking cessation: It's not in the trying, but in the succeeding. Tobacco control. October 20, 2008 2009;18(1):43-46.

24. Brose LS, West R, McDermott MS, Fidler JA, Croghan E, McEwen A. What makes for an effective stop-smoking service? Thorax. June 27, 20112011.

25. Brose LS, McEwen A, West R. Does it matter who you see to help you stop smoking? Shortterm quit rates across specialist Stop Smoking Practitioners in England. Addiction (Abingdon, England). 2012;107(11):2029-2036.

26. Zhou X, Nonnemaker J, Sherrill B, Gilsenan AW, Coste F, West R. Attempts to quit smoking and relapse: factors associated with success or failure from the ATTEMPT cohort study. Addict Behav. Apr 2009;34(4):365-373.

27. Borland R, Li L, Driezen P, et al. Cessation assistance reported by smokers in 15 countries participating in the International Tobacco Control (ITC) policy evaluation surveys. Addiction (Abingdon, England). 2012;107(1):197-205.

28. Fidler J, Shahab L, West O, et al. 'The Smoking Toolkit Study': A national study of smoking and smoking cessation in England. BMC public health. 2011;11(1):479.

29. The Smoking Toolkit Study. www.smokinginengland.info. Accessed 16 April 2011. 
30. Wong SL, Shields M, Leatherdale S, Malaison E, Hammond D. Assessment of validity of selfreported smoking status. Health Rep. Mar 2012;23(1):47-53.

31. West R, Zatonski W, Przewozniak K, Jarvis MJ. Can we trust national smoking prevalence figures? Discrepancies between biochemically assessed and self-reported smoking rates in three countries. Cancer Epidemiol Biomarkers Prev. Apr 2007;16(4):820-822.

32. Fidler JA, Shahab L, West R. Strength of urges to smoke as a measure of severity of cigarette dependence: comparison with the Fagerstrom Test for Nicotine Dependence and its components. Addiction (Abingdon, England). 2011;106(3):631-638.

33. Fiore MC, Jaén CR, Baker TB, et al. Treating tobacco use and dependence: 2008 update. Rockville, MD: US Department of Health and Human Services; 2008.

34. Hughes JR, Shiffman S, Callas P, Zhang J. A meta-analysis of the efficacy of over-the-counter nicotine replacement. Tobacco control. 2003;12(1):21-27.

35. Kouvonen A, Kivimäki M, Oksanen T, et al. Implementation of Workplace-Based Smoking Cessation Support Activities and Smoking Cessation Among Employees: The Finnish Public Sector Study. American journal of public health. 2012/06/01 2012 May 17 [Epub ahead of print]:e1-e7.

36. Nonnemaker J, Hersey J, Homsi G, et al. Self-reported exposure to policy and environmental influences on smoking cessation and relapse: a 2-year longitudinal population-based study. Int J Environ Res Public Health. Sep 2011;8(9):3591-3608.

37. Boutou AK, Tsiata EA, Pataka A, Kontou PK, Pitsiou GG, Argyropoulou P. Smoking cessation in clinical practice: predictors of six-month continuous abstinence in a sample of Greek smokers. Primary Care Respiratory Journal. 2008;17(1):32-38. 
38. Zhu S, Melcer T, Sun J, Rosbrook B, Pierce JP. Smoking cessation with and without assistance: a population-based analysis. American journal of preventive medicine. May $2000 ; 18(4): 305-311$.

39. Taggar J, Coleman T, Lewis S, Szatkowski L. The impact of the Quality and Outcomes Framework (QOF) on the recording of smoking targets in primary care medical records: cross-sectional analyses from The Health Improvement Network (THIN) database. BMC public health. 2012;12(1):329.

40. Lawrence D, Mitrou F, Zubrick SR. Non-specific psychological distress, smoking status and smoking cessation: United States National Health Interview Survey 2005. BMC public health. 2011;11:256

41. Vangeli E, Stapleton J, Smit ES, Borland R, West R. Predictors of attempts to stop smoking and their success in adult general population samples: A systematic review. Addiction (Abingdon, England). 2011;106(12):2110-2121.

42. Berg CJ, An LC, Kirch M, et al. Failure to report attempts to quit smoking. Addict Behav. 2010;35(10):900-904.

43. West R, Hajek P, Stead L, Stapleton J. Outcome criteria in smoking cessation trials: proposal for a common standard. Addiction (Abingdon, England). Mar 2005;100(3):299-303.

44. Kotz D, Fidler J, West R. Very low rate and light smokers: smoking patterns and cessationrelated behaviour in England, 2006-2011. Addiction (Abingdon, England). 2012;107(5):9951002. 
Table 1: Sample characteristics $(\mathrm{N}=10,335)$

Age, mean (SD)

$39.5(15.6)$

Female sex

$54.0(5,576)$

Social grade

$A B$

$10.7(1,105)$

C1

$22.9(2,371)$

$\mathrm{C} 2$

$22.7(2,351)$

D

$18.8(1,945)$

$E$

$24.8(2,563)$

Current non-smokers

$13.6(1,403)$

Time since last quit attempt started

$$
<=1 \text { to } 26 \text { weeks }
$$$$
63.0(6,510)
$$

26 to 52 weeks

$37.0(3,825)$

Number of quit attempts in the past year

1

$64.5(6,661)$

2

$21.9(2,264)$

3

$7.6(783)$

4 or more

$6.1(627)$

Use of smoking cessation treatments

Medication on prescription + specialist behavioural support

$2.0(204)$

Medication on prescription + brief advice

$16.5(1,706)$

NRT bought over-the-counter

$30.3(3,128)$

None of the above

$3.0(1.3)$

Time spent with urges to smoke, mean (SD)

$2.0(1.2)$

Strength of urges to smoke, mean (SD)

Figures are presented as percentage $(\mathrm{N})$, unless stated otherwise. Medication on prescription included nicotine replacement therapy (NRT), varenicline or bupropion. Time spent with urges to smoke: 1 (not at all) to 6 (all the time). Strength of urges to smoke: 0 (no urges) to 5 (extremely strong urges). 
Table 2: Associations between characteristics of the sample and use of smoking cessation treatments

\begin{tabular}{|c|c|c|c|c|c|}
\hline Variable & $\begin{array}{c}\text { Medication on } \\
\text { prescription + specialist } \\
\text { behavioural support } \\
(N=204)\end{array}$ & $\begin{array}{c}\text { Medication on } \\
\text { prescription }+ \text { brief } \\
\text { advice } \\
(\mathrm{N}=1,706) \\
\end{array}$ & $\begin{array}{l}\text { NRT bought over-the- } \\
\text { counter } \\
(\mathrm{N}=3,128)\end{array}$ & $\begin{array}{l}\text { None of the other } \\
\qquad(N=5,297)\end{array}$ & $\mathbf{P}$ \\
\hline Age, mean (SD) & $46.5(14.1)$ & $43.3(14.8)$ & $40.8(15.0)$ & $37.2(15.9)$ & $* * *$ \\
\hline \multicolumn{6}{|l|}{ Sex } \\
\hline Male & $34.3(70)$ & $42.4(724)$ & $45.4(1,424)$ & $48.0(2,541)$ & $* * *$ \\
\hline Female & $65.7(134)$ & $57.6(982)$ & $54.5(1,704)$ & $52.0(2,756)$ & \\
\hline \multicolumn{6}{|l|}{ Social grade } \\
\hline$A B$ & $13.7(28)$ & $9.4(160)$ & $11.1(348)$ & $10.7(569)$ & $* *$ \\
\hline $\mathrm{C} 1$ & $21.1(43)$ & $20.1(343)$ & $23.4(732)$ & $23.7(1,253)$ & \\
\hline $\mathrm{C} 2$ & $25.0(51)$ & 23.4 (399) & $21.5(671)$ & $23.2(1,230)$ & \\
\hline $\mathrm{D}$ & $14.2(29)$ & $19.5(332)$ & $18.0(564)$ & $19.3(1,020)$ & \\
\hline $\mathrm{E}$ & $26.0(53)$ & $27.7(472)$ & $26.0(813)$ & $23.1(1,225)$ & \\
\hline \multicolumn{6}{|l|}{ Time since quit attempt started } \\
\hline$<=1$ to 26 weeks & $53.4(109)$ & $60.3(1,028)$ & $63.4(1,984)$ & $64.0(3,389)$ & $* *$ \\
\hline 26 to 52 weeks & $45.6(95)$ & $39.7(678)$ & $36.6(1,144)$ & $36.0(1,908)$ & \\
\hline Number of quit attempts in the past year & & & & & n.s. \\
\hline 1 & $64.7(132)$ & $67.6(1,153)$ & $63.0(1,970)$ & $64.3(3,406)$ & \\
\hline 2 & $22.1(45)$ & $20.9(356)$ & $22.5(703)$ & $21.9(1,160)$ & \\
\hline 3 & $6.9(14)$ & $6.0(103)$ & $8.5(266)$ & $7.6(400)$ & \\
\hline 4 or more & $6.4(13)$ & $5.5(94)$ & $6.0(189)$ & $6.2(331)$ & \\
\hline Time spent with urges to smoke, mean (SD) & $3.3(1.3)$ & $3.2(1.4)$ & $3.2(1.2)$ & $2.8(1.2)$ & $* * *$ \\
\hline Strength of urges to smoke, mean (SD) & $2.3(1.3)$ & $2.2(1.2)$ & $2.2(1.1)$ & $1.8(1.1)$ & $* * *$ \\
\hline
\end{tabular}


Table 3: Differences in unadjusted measurements of strength of urges to smoke in smokers versus non-smokers, stratified by method of quitting Method of quitting

\section{Mean (SD) strength}

of urges to smoke

in smokers

Medication on prescription + specialist behavioural support

Medication on prescription + brief advice

NRT bought over-the-counter

None of the above

(N)

(165)

$(1,447)$

59 (1.10)

$(2,806)$

$(4,514)$

$2.43(1.05)$

$2.33(1.03)$

None of the above $\quad 2.02(1.03) \quad(4,514) \quad(783)$

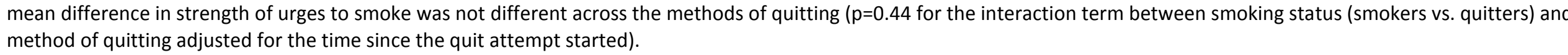

of urges to smoke in
Mean (SD) strength
Mean difference

$(95 \% \mathrm{Cl})$ in strength

of urges to smoke

(39) $1.31(1.66) \quad 1.29(0.73-1.85)$

(259) $\quad 1.05(1.31) \quad 1.38(1.21-1.55)$

$\begin{array}{lll}(259) & 1.05(1.31) & 1.38(1.21-1.55) \\ (322) & 1.06(1.24) & 1.28(1.14-1.42)\end{array}$

$0.76(1.16)$

$1.26(1.17-1.35)$ 
Table 4: Unadjusted and adjusted odds of self-reported non-smoking in the full sample and in the two subsamples of respondents who started their quit attempt less/more than 6 months ago

Smoking cessation treatment

OR $(95 \% \mathrm{Cl})$

\section{Full sample $(\mathbf{N}=\mathbf{1 0 , 3 3 5 )}$}

Medication on prescription + specialist behavioural support $(\mathrm{N}=204)$

Medication on prescription + brief advice $(\mathrm{N}=1,706)$

Model 1

Model 2

Model 3

Model 4

$1.36(0.95-1.95)$

$1.47(1.02-2.11)$

$2.97(1.93-4.59)$

$1.59(1.32-1.91)$

$1.61(1.33-1.94)$

NRT bought over-the-counter $(\mathrm{N}=3,128)$

None of the above (reference) $(\mathrm{N}=5,297)$

\section{Subsample: quit attempt started $<6$ months $(\mathrm{N}=6,510)$}

Medication on prescription + specialist behavioural support $(\mathrm{N}=109)$

Medication on prescription + brief advice $(\mathrm{N}=1,028)$

NRT bought over-the-counter $(\mathrm{N}=1,984)$

None of the above (reference) $(\mathrm{N}=3,389)$

Subsample: quit attempt started $>6$ months $(\mathrm{N}=3,825)$

Medication on prescription + specialist behavioural support $(\mathrm{N}=95)$

Medication on prescription + brief advice $(\mathrm{N}=678$

NRT bought over-the-counter $(\mathrm{N}=1,144)$

None of the above (reference) $(\mathrm{N}=1,908)$

0.95 (0.81-1.12)

$0.66(0.58-0.76)$

$0.63(0.55-0.74)$

1

$1.45(0.90-2.34)$
$1.21(1.00-1.46)$
$0.60(0.60-0.84)$

$\begin{array}{ll}1.27(0.74-2.17) & 1.14(0.66-1.98) \\ 0.78(0.60-1.01) & 0.74(0.57-0.98) \\ 0.59(0.46-0.75) & 0.56(0.44-0.71)\end{array}$

$$
\begin{aligned}
& 1.83(1.12-3.01) \\
& 1.20(0.98-1.46) \\
& 0.68(0.57-0.82)
\end{aligned}
$$

$$
1
$$

$$
\begin{gathered}
3.80(2.17-6.67) \\
1.78(1.42-2.23) \\
0.97(0.80-1.00) \\
1
\end{gathered}
$$$$
0.96(0.81-1.13)
$$

(t)

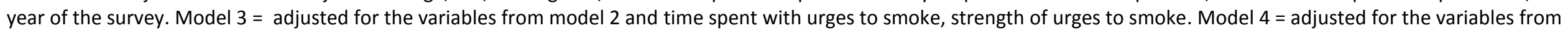
model 2 and the interaction terms time since last quit attempt started * time spent with urges, time since last quit attempt started * strength of urges to smoke. Medication on prescription included nicotine replacement therapy (NRT), varenicline or bupropion. OR $=$ odds ratio, $95 \% \mathrm{Cl}=95 \%$ confidence interval around OR. 
Supplementary Table E1: Percentage $(95 \% \mathrm{Cl})$ non-smokers in each treatment condition stratified by strength of urges to smoke in the full sample $(\mathrm{N}=10,335)$

\begin{tabular}{|c|c|c|}
\hline Smoking cessation treatment & $\begin{array}{c}\text { Strength of urges to } \\
\text { smoke }^{\ddagger}\end{array}$ & Non-smoker \\
\hline \multirow[t]{2}{*}{ Medication on prescription + specialist behavioural support } & $\begin{array}{c}\text { Low } \\
(\mathrm{N}=117)\end{array}$ & $25.6(17.7-33.6)$ \\
\hline & $\begin{array}{l}\text { High } \\
(\mathrm{N}=87)\end{array}$ & $10.3(3.9-16.7)$ \\
\hline \multirow[t]{2}{*}{ Medication on prescription + brief advice } & $\begin{array}{c}\text { Low } \\
(\mathrm{N}=1,030)\end{array}$ & $21.1(18.6-23.6)$ \\
\hline & $\begin{array}{c}\text { High } \\
(\mathrm{N}=676)\end{array}$ & $6.2(4.4-8.0)$ \\
\hline \multirow[t]{2}{*}{ NRT bought over-the-counter } & $\begin{array}{c}\text { Low } \\
(\mathrm{N}=2,033)\end{array}$ & $13.8(12.3-15.3)$ \\
\hline & $\begin{array}{c}\text { High } \\
(\mathrm{N}=1,095)\end{array}$ & $3.8(2.7-5.0)$ \\
\hline \multirow[t]{2}{*}{ None of the above } & $\begin{array}{c}\text { Low } \\
(\mathrm{N}=4,011)\end{array}$ & $17.7(16.5-18.6)$ \\
\hline & $\begin{array}{c}\text { High } \\
(\mathrm{N}=1,286)\end{array}$ & $5.8(4.5-7.0)$ \\
\hline
\end{tabular}

${ }^{\ddagger}$ Split by median: low strength of urges to smoke = scores 0 to 2 , high = scores 3 to $5.95 \% \mathrm{Cl}=95 \%$ confidence interval. 